\title{
Correction Method Based on KI-VPA Model for Changes in Vibratory Perception Caused by Adaptation
}

\author{
Yuki Mori $^{1}$, Takayuki Tanaka ${ }^{2}$, and Shun'ichi Kaneko ${ }^{2}$ \\ ${ }^{1}$ RIKEN, Nagoya, Aichi, Japan \\ mori@nagoya.riken.jp \\ ${ }^{2}$ Hokkaido University, Sapporo, Hokkaido, Japan \\ \{ttanaka, kaneko\} @ssi.ist.hokudai.ac.jp
}

\begin{abstract}
This paper describes a method for correcting differences in human vibratory perception caused by sensory adaptation. Humans feel a vibrational strength when a vibrating device is held in the hand. However when the vibrational frequency is changed, human perception of the new frequency is affected by the vibrational frequency experienced before the change. This is called sensory adaptation. The Katagiri-Aida model-based vibratory perception adaptation (KI-VPA) mode can estimate changes in vibratory perception caused bt adaptation. We have developed a correction method on basis of the KI-VPA model and tested the method on ten human subjects. The results indicate that the proposed correction method reduced the effects of adaptive changes to vibratory perception.
\end{abstract}

Keywords: Vibration, Vibratory perception, Tactile sense, Vibration alert interface.

\section{Introduction}

In recent years, a number of studies on information presentation through touch have been conducted; examples include tactile display, sensory substitution and virtual reality $[1,2]$. We have been developing a vibration alert interface (VAI), which is a handheld vibrating that conveys information such as distance and degree of risk by changing the vibrational frequency. The current studies of informational presentation mainly focused on the configuration of the device and its vibrational patterns for conveying precise information. However, to convey precise information, it needs to consider disturbance which effect vibration perception. Yao, H.S. et al. have examined relation between weight of vibrating device and perceived vibration strength [3]. Morioka et al. have reported difference in perception threshold caused by vibration direction [4]. Thus it is known that vibration sensation varies with various factors other than vibration amplitude and frequency. The paper by J. F. Hahn has reported vibrotactile adaptation and recovery [5], M.Tommerdahl et al. have demonstrated frequency discriminative capacity after adaptation to vibration stimulation [6]. Our focus has been on adaptation to vibratory perception. We have experimentally found that human vibratory perceptions change by adapting to the sensations caused by 
vibrations. In particular subjects feel different vibrational strengths from the same frequency when that frequency is the result of a change from other frequencies. We have also proposed the Katagiri-Aida model-based vibratory perception adaptation (KI-VPA) model to predict changes in vibratory perception caused by changes in frequency. The model correctly shows changes in human vibratory perception.

In this study, we show that the KI-VPA model can be used as the basis for correcting changes in vibratory perception that are caused by adaptation. The KI-VPA model provides correction frequency on the basis of the vibratory perception to be conveyed by VAI; the model also considers duration of the frequency experienced before the change. In human tests, in accordance with the frequency before the change, subjects felt a precise vibrational strength from the correction frequency after the change.

This paper is organized as follows. Section 2 describes an experiment that examines how vibratory perception is affected by the frequency before the change. Section 3 explains the modeling of the change in vibratory perception described in Section 2. Section 3 also describes our method for correcting this change. Section 4 presents results obtained from experiments with human subjects that verify the effectiveness of the correction method proposed in Section 3. Section 5 states conclusions drawn from this work.

\section{Changes in Vibratory Perception Caused by Changes in Vibrational Frequency}

\subsection{Experimental Method}

Many devices use vibrations to convey information or issue warnings. When a user feels a vibration from a device, the user interprets signals on the basis of the vibrational strength. However, even when the vibrational frequency is the same, the user can feel the differences in vibrational strength. The user can misinterpret signals because of adaptation; when the vibrational frequency changes, the frequency before the change affects the user's perception of the vibrations after the change. In this section, we discuss experiments that show how vibratory perceptions after a frequency change are affected by the frequency before the change.

When that device's user feels vibration, user read information from vibration strength. Although vibration frequency is the same, user can feel different vibration strength. One of the causes is the adaptation to the vibration. When the vibration frequency changes, the frequency before the change affects vibration perception user feels after the change. Therefore this section examine experimentally how vibratory perception after the shift changes with the frequency before the shift.

Experiments were conducted to examine whether vibratory perceptions are changed by adaptation. In the experiments, vibrations were conveyed to subjects using VAI shown in Fig. 1. VAI had a diameter of $4 \mathrm{~cm}$ and a length of $10 \mathrm{~cm}$. A vibration motor was mounted inside the device. The experimental procedure was as follows: 


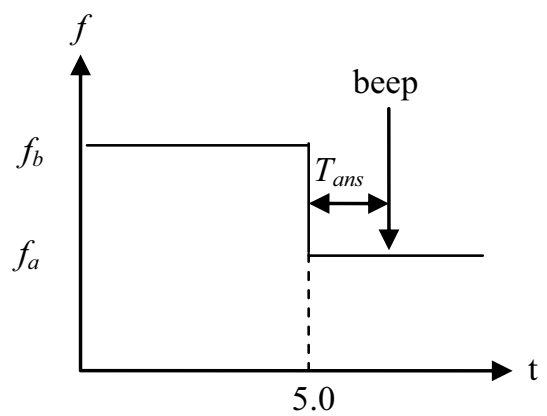

Fig. 1. Timing of the step change in frequency from the initial value $f_{b}$ to the final value $f_{a}$. After a delay of $T_{a n s} \mathrm{~s}$, the device beeps, signaling the user to record the perceived final frequency. On the abscissa, time is in seconds.

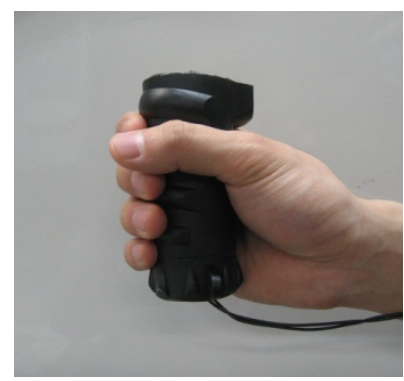

Fig. 2. Vibration alert interface. Length is $10 \mathrm{~cm}$, diameter is $3.5 \mathrm{~cm}$. A compact DC motor is mounted in the device.

1. Subjects grasped VAI. They were instructed to associate a relative vibrational intensity of 10 with VAI vibrations at $70 \mathrm{~Hz}$ and an intensity of 20 with vibrations at $150 \mathrm{~Hz}$.

2. VAI vibrated for $5 \mathrm{~s}$ at one of these frequencies: 70, 90, 110, 130, or $150 \mathrm{~Hz}$. This frequency was the one before the change, $f_{b}$, and its duration was $T_{f b}$.

3. Then, the frequency $f_{b}$ was changed via a step function. The frequency after the change was represented as $f_{a}$ and its duration was $T_{f a}$.

4. It beeped after another $T_{\text {ans }}[\mathrm{s}]$. The values used for $T_{\text {ans }}$ were $1.0,2.0$, or $3.0 \mathrm{~s}$. At the beep, subjects reported their perceived vibrational intensities relative to the standard values 10 and 20. These reported vibrational strengths were represented by $P_{v a}$.

The timings of events in steps 2-4 are illustrated in Fig. 2. In this experiment, $T_{f b}$ and $T_{f a}$ were both $5 \mathrm{~s}$. Each subject repeated steps 1-4 20 times at five values of $f_{b}$ and five values of $f_{a}$. Moreover, they repeated the steps three times using each of the allowed values for the interval to beep, $T_{a n s}$. The test involved 10 subjects, both male and female, with an average age of 25.5 years. During the experiments, subjects wore eye masks and heard white noise through headphones to ensure that they were focused solely on their tactile senses.

\subsection{Experimental Result}

The experimental results are shown in Fig. 3. The plots in the figure show how the perceived vibrational strength after the change $\left(P_{v a}\right)$ relates to the frequency before the change $\left(f_{b}\right)$. In Figs. 3(a), 3(b), and 3(c), the frequency after the change $\left(f_{a}\right)$ is treated as a parameter. Each panel in Fig. 3 is for a different interval between the change and the beep. Figures 3(a), 3(b), and 3(c) had intervals of 1, 2, and 3 s, respectively. Each point in the figure is an average over the ten subjects, and the lines are linear approximations to the points. 


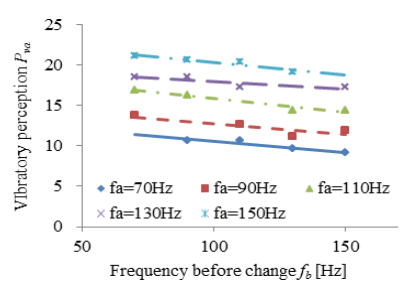

(a) $T_{\text {ans }}=1.0 \mathrm{~s}$

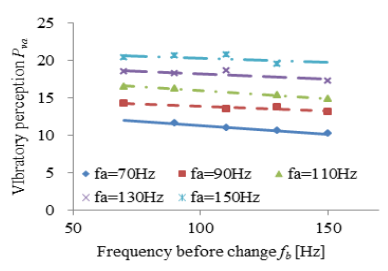

(b) $T_{\text {ans }}=2.0 \mathrm{~s}$

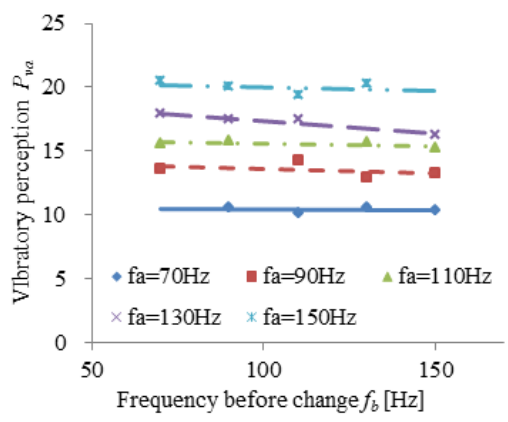

(c) $T_{\text {ans }}=3.0 \mathrm{~s}$

Fig. 3. Change in vibratory perception caused by adaptation. Points are experimental values averaged over 10 subjects. Lines are linear fits to the points. $T_{a n s}$ of (a) $1 \mathrm{~s}$, (b) $2 \mathrm{~s}$, (c) $3 \mathrm{~s}$.

Table 1. Slopes of the lines in Fig. 3 for $P_{v a}$ vs $f_{b}$

\begin{tabular}{|r|r|r|r|}
\hline$f_{b}[\mathrm{~Hz}]$ & $T_{\text {ans }}=1.0$ & $T_{\text {ans }}=2.0$ & $T_{\text {ans }}=3.0$ \\
\hline 70 & -0.029 & -0.023 & -0.001 \\
\hline 90 & -0.027 & -0.012 & -0.007 \\
\hline 110 & -0.034 & -0.021 & -0.004 \\
\hline 130 & -0.018 & -0.015 & -0.020 \\
\hline 150 & -0.031 & -0.012 & -0.007 \\
\hline Average & -0.028 & -0.017 & -0.008 \\
\hline
\end{tabular}

Since each line in each panel of Fig. 3 is at one final frequency $f_{a}$, each line should be horizontal if the perceptions of the subjects were consistent. However few lines in Fig. 3 are close to horizontal, and all have negative slopes. The slopes are tabulated in Table 1 . The negative slopes indicate that higher the initial frequency $f_{b}$, weaker is the perceived vibration after the frequency change. This occurred irrespective of the final frequency $f_{a}$ being greater or lesser than the initial frequency $f_{b}$. This implies that the perceived vibrational strengths depend on not only final frequency $f_{a}$ but also the initial frequency $f_{b}$; we interpret this as evidence for adaptive behavior. 
Further note that the lines become more horizontal from Fig. 3(a) to Fig. 3(c), i.e., with increase in the interval of times after frequency changes. In Table 1, the slopes at $T_{a n s}=1.0 \mathrm{~s}$ (Fig. 3(a)) are larger than those at $T_{a n s}=3.0 \mathrm{~s}$ (Fig. 3(c)). This implies that the influence of $f_{b}$ on $P_{v a}$ is large immediately after a frequency change but the influence decreases with time.

These experiments demonstrate that vibratory perception is affected not only by the current frequency but also by earlier frequencies. If the frequency before the change was low, subjects perceived strong vibrations after the change. But if the frequency before the change was high, subjects perceived weak vibrations after the change. Moreover, the influence of changes in frequency on vibratory perception decreased with time.

\section{Correction Method for Change in Vibratory Perception by Adaptation}

\subsection{Adaptation Model}

In this section, we model adaptive changes in vibratory perception using the KI-VPA model [7], which is based on the KI model shown in Fig. 3 [8]. The KI model consists of excitatory and inhibitory neuron firings and is represented as follows:

$$
u_{ \pm}(t)= \pm \frac{1}{2} Q_{0} \frac{R \alpha_{ \pm}}{\sqrt{\pi t}} \exp \left(-\frac{\beta_{ \pm}}{\alpha_{ \pm}^{2}} t\right)
$$

where $u$ is the potential for both excitatory (+) and inhibitory (-) responses, $t$ is time, while $\alpha$ and $\beta$ are parameters determined experimentally. Quantities $Q_{0}$ and $R$ are constants; following [2], we set $Q_{0}=1.0$ and $R=2.0$. Figure 4 displays the potential outputs of excitatory and inhibitory neurons. Excitatory neurons fire a positive pulse (Fig. 4(a)), while inhibitory neurons fire a negative pulse (Fig. 4(b)). The output from the KI-VPA model is represented as follows:

$$
u(t)=a \sum_{i=1}^{N}\left[u_{+}\left(t-i \frac{1}{f(t)}+\tau\right)+u_{-}\left(t-i \frac{1}{f(t)}\right)\right]+b
$$

where $\tau$ is the difference in firing times between excitatory and inhibitory neurons. An excitatory neuron fires when a vibrational stimulus is inputted, while an inhibitory neuron fires after a time lag. In the above equation, $f(t)$ is the vibrational frequency, and $a$ and $b$ are constants determined experimentally. To minimize the error between the model and the experimental results in Fig. 3, we set $a=1.25, b=4.42, \alpha_{+}=1.5$, $\alpha_{-}=0.8, \beta_{+} / \alpha_{+}{ }^{2}=3.6$, and $\beta_{-} / \alpha_{-}{ }^{2}=2.0$. Figure. 5 shows the change in output of the KI-VPA model when frequency change to $110 \mathrm{~Hz}$ from $70-150 \mathrm{~Hz}$. After $5 \mathrm{~s}$, it is confirmed that vibratory perceptions are different even though frequency after the change are the same. 


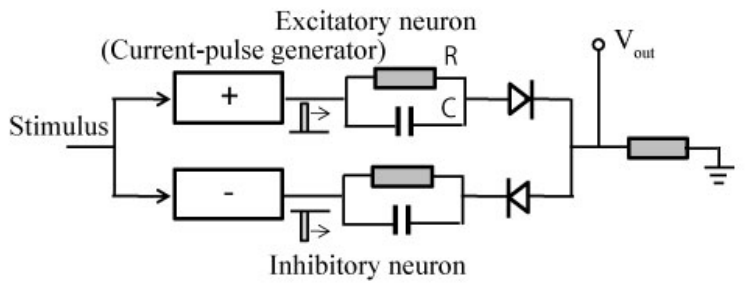

Fig. 4. Equivalent circuit for the KI model

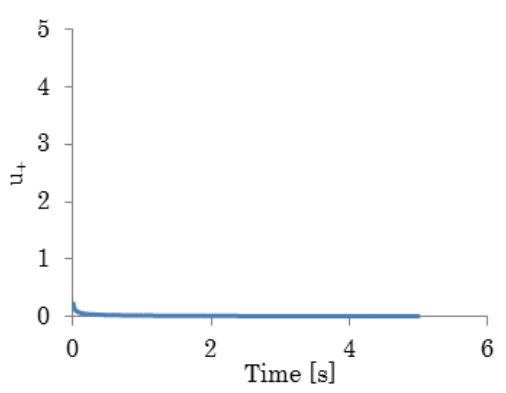

(a) Excitatory neuron

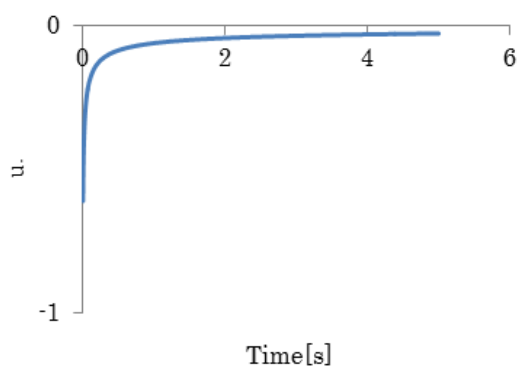

(b) Inhibitory neuron

Fig. 5. Pulse forms created by firing of two types of neurons

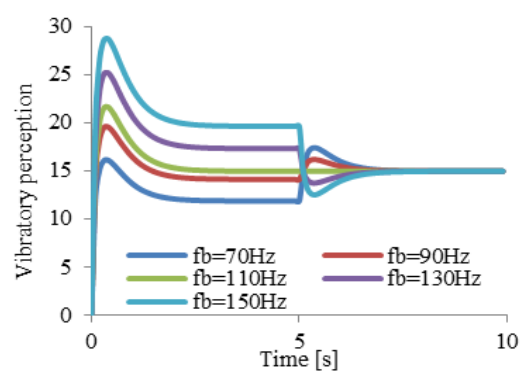

Fig. 6. Output of KI-VPA model when frequencies change to $110 \mathrm{~Hz}$ from $70-150 \mathrm{~Hz}$

The outputs from the KI-VPA model are shown in Fig. 6. In the figure, the lines are from the KI-VPA model, and the points are experimental data from Fig. 3. The figure shows that the KI-VPA model captures changes in vibratory perception with high accuracy.

\subsection{Correction Method}

With vibrational frequency as the input, the KI-VPA model outputs the vibratory perception felt by a user. Therefore, if the relation between the frequency and 
vibratory perception is known, VAI enables the user to feel the desired vibration by adjusting the vibrational frequency depending on the frequency before the change. We perform this correction using the schematic diagram shown in Fig. 6.

The KI-VPA model establishes a linear relation among the vibratory perceptions felt by subjects after the change $\left(P_{v a}\right)$, the frequency before the change $\left(f_{b}\right)$, and the duration $\left(T_{f b}\right)$ :

$$
P_{v a}=g_{1} f_{b}+g_{2} T_{f b}+g_{3} f_{a}+g_{4}
$$

where $g_{l} \sim g_{4}$ are non-linearly functions of $T_{a n s}$. Especially $g_{4}$ is a function showing the relationship between $P_{v a}$ and $T_{a n s}$. $T_{a n s}$ is time to report after it beeped. If $T_{a n s}$ is constant, $P_{v a}$ is given by

$$
P_{v a}=a_{1} f_{b}+a_{2} T_{f b}+a_{3} f_{a}+a_{4}
$$

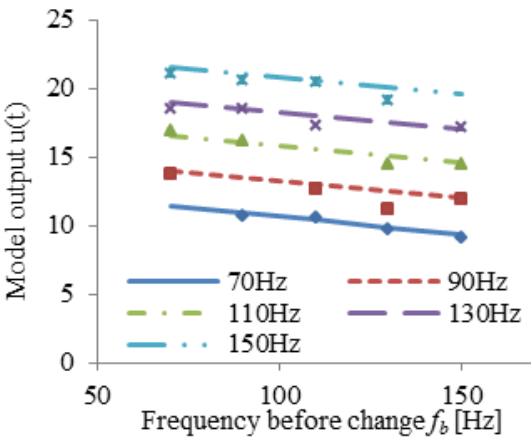

(a) $T_{\text {ans }}=1.0 \mathrm{~s}$

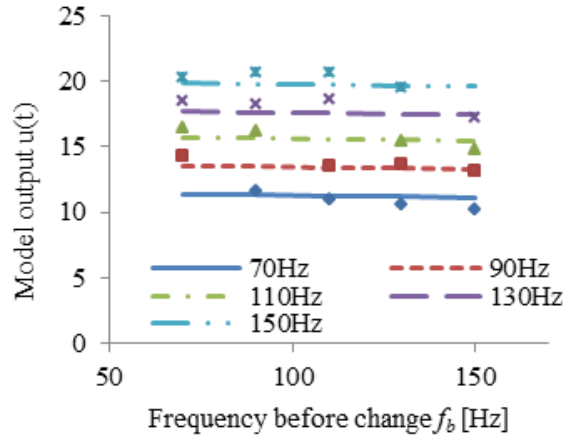

(b) $T_{\text {ans }}=2.0 \mathrm{~s}$

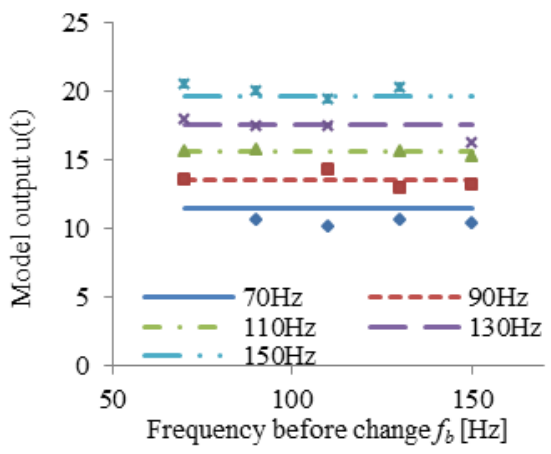

c) $T_{\text {ans }}=3.0$

Fig. 7. Output of the K I-VPA model (lines) compared with the experimental data in Fig. 3 (points). $T_{a n s}$ are the same as in Fig. 3. 
where $a_{l}-a_{4}$ are constants. Therefore, the correction frequency $f_{a}$ ' that eliminates the effects of adaptation is calculated in the KI-VPA model by

$$
f_{a}^{\prime}=\frac{1}{a_{3}}\left(P_{v a}-a_{1} f_{b}-a_{2} T_{f b}-a_{4}\right)
$$

This equation provides the frequency that eliminates the influence of adaptation. It allows the VAI device to convey $P_{v a}$ by vibrating at $f_{a}$, after VAI has vibrated at an initial frequency $f_{b}$ over a duration $T_{f b}$.

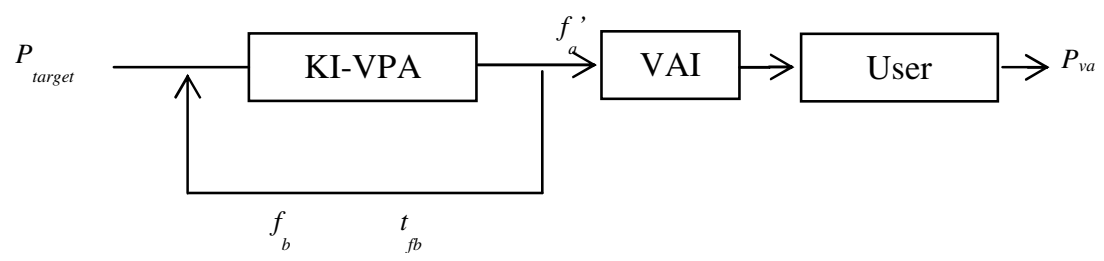

Fig. 8. Schematic of the correction method based on the KI-VPA model used in the VAI device to correct perceived vibrations for effects of adaptation

\section{Correction Experiment}

Correction experiments were performed to confirm the effectiveness of the correction formula presented in Section 3. The subjects were the same 10 people who participated in the previous experiment. The experimental method was the same as that used in Section 2.1, except that step 3 was changed to the following:

3. The frequency $f_{b}$ was changed via a step function. This new frequency was the correction frequency $f_{a}$ ' that eliminates changes in vibratory perception due to adaptation as calculated by the KI-VPA model.

In the correction experiments, VAI vibrated at the correction frequency after it vibrated at $f_{b}$ for $5 \mathrm{~s}$. In the experiments described in Section 2, the time to report $T_{\text {ans }}$ were 1.0, 2.0, and $3.0 \mathrm{~s}$. However, in the correction experiments, we reduced $T_{a n s}$ to $0.5,1.0$, and $1.5 \mathrm{~s}$ because the results in Section 2.2 show that adaptation has greater effects on vibratory perception immediately after the change in frequency. $P_{v a}$ in eq. (4) is obtained by calculating average value of $P_{v a}$ on $f_{b}=f_{a}$ in Figs. 3(a), 3(b), and 3(c).

The results from the correction experiments are shown in Fig. 8. In Fig. 3, the data were represented by lines with negative slopes because vibratory perceptions were changed by adaptation. However, the lines in Fig. 8 are nearly horizontal, i.e., with the correction model, VAI conveys precise vibratory perceptions without adaptive effects. Further, the correction model is effective at all times $T_{\text {ans }}$ since all the approximated lines in Figs. 8(a), 8(b), and 8(c) are nearly horizontal. The slope of each line is shown in Table 2. Compared to the data in Table 1, the values in Table 2 are lower, confirming that changes in vibratory perception did not occur in the correction experiments. 


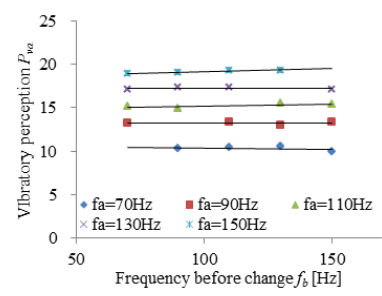

(a) $T_{\text {ans }}=0.5 \mathrm{~s}$

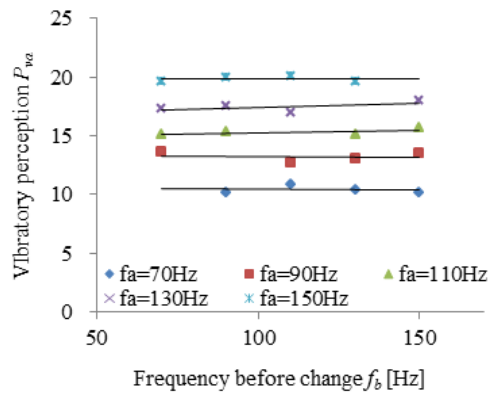

(c) $T_{\text {ans }}=1.5 \mathrm{~s}$

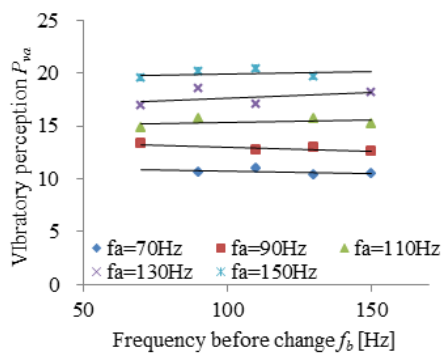

(b) $T_{\text {ans }}=1$.

Fig. 9. Results from the correction experiments. Points are experimental data and lines are linear fits to the points. Times to report of (a) $0.5 \mathrm{~s}$, (b) $1.0 \mathrm{~s}$, (c) $1.5 \mathrm{~s}$.

Table 2. Slopes of lines in Fig. 8 from the correction experiments. MAV is the mean of the absolute values.

\begin{tabular}{|r|r|r|r|}
\hline \multicolumn{1}{|c|}{$f_{b}[\mathrm{~Hz}]$} & $T_{\text {ans }}=0.5$ & $T_{\text {ans }}=1.0$ & $T_{\text {ans }}=1.5$ \\
\hline 70 & 0.0035 & -0.0045 & -0.0025 \\
\hline 90 & 0.0000 & -0.0079 & -0.0019 \\
\hline 110 & 0.0052 & 0.0040 & 0.0045 \\
\hline 130 & -0.0010 & 0.0101 & 0.0073 \\
\hline 150 & 0.0075 & 0.0040 & 0.0005 \\
\hline MAV & 0.0034 & 0.0061 & 0.0033 \\
\hline
\end{tabular}

\section{Conclusions}

In this study, we used human subjects to experimentally measure changes in vibratory perception caused by adaptation. Then, we modeled those changes in vibratory perception using the KI-VPA model. The model provides a correction formula that eliminates changes in vibratory perception due to adaptation. We also performed additional experiments to test the correction formula; the experimental results verified 
the effectiveness of the correction model. In particular, correction frequencies calculated by the model can cancel the influence of adaptation.

To date, information presentation devices using vibration have not been able to convey precise information using changes in vibrational frequency, because vibratory perception is changed by adaptation. However, owing to this study, such devices should be able to convey precise information by vibrating at the corrected frequencies provided by our model.

\section{References}

1. Tsukada, K., Yasumura, M.: ActiveBelt: Belt-Type Wearable Tactile Display for Directional Navigation. In: Mynatt, E.D., Siio, I. (eds.) UbiComp 2004. LNCS, vol. 3205, pp. 384399. Springer, Heidelberg (2004)

2. Jones, L.A., Lockyer, B., Piateski, E.: Tactile display and vibrotactile pattern recognition on the torso. Advanced Robotics 20(12), 1359-1374 (2006)

3. Yao, H.S., Grant, D., Cruz, M.: Perceived Vibration Strength in Mobile Devices: the Effect of Weight and Frequency. IEEE Transaction on Haptics (2009)

4. Morioka, M., Griffin, M.J.: Magnitude-dependence of equivalent comfort contours for foreand-art, lateral and vertical hand-transmitted vibration. Journal of Sound and Vibration 295, 633-648 (2006)

5. Hahn, J.F.: Vibrotactile Adaptation and Recovery Measured by Two Methods. Jounal of Experimental Psychology 71(5), 655-658 (1966)

6. Tommerdahl, M., Hester, K.D., Felix, E.R., Hollins, M., Favorov, O.V., Quibrera, P.M., Whitsel, B.L.: Human vibrotactile frequency discriminative capacity after adaptation to 25 $\mathrm{Hz}$ or $200 \mathrm{~Hz}$ stimulation. Brain Research 1057, 1-9 (2005)

7. Mori, Y., Tanaka, T., Kaneko, S.: Design of Vibration Alert Interface Based on Tactile Adaptation Model to Vibration Stimulation. In: Smith, M.J., Salvendy, G. (eds.) HCII 2011, Part I. LNCS, vol. 6771, pp. 462-469. Springer, Heidelberg (2011)

8. Katagiri, Y., Aida, K.: Simulated nonlinear dynamics of laterally interactive arrayed neurons. In: Optomechatronic Technologies 2008 SPIE Proceedings (2008) 